\title{
Abordagem para o desenvolvimento de disciplinas sobre informação em saúde em cursos de graduação
}

Approach to develop health information subjects in undergraduate courses

Abordaje para el desarrollo de asignaturas sobre información en salud en cursos de pregrado

Recebido: 26/01/2022 | Revisado: 30/01/2022 | Aceito: 01/02/2022 | Publicado: 03/02/2022

Maria Cristiane Barbosa Galvão

ORCID: https://orcid.org/0000-0003-3971-5743

Universidade de São Paulo, Brasil

E-mail:mgalvao@usp.br

Brianda de Oliveira Ordonho Sígolo

ORCID: https://orcid.org/0000-0002-7886-6985

Universidade Estadual Paulista Júlio de Mesquita Filho, Brasil

E-mail: brianda@usp.br

Fabrício Amadeu Gualdani

ORCID: https://orcid.org/0000-0001-7426-0831

Universidade Estadual Paulista Júlio de Mesquita Filho, Brasil

E-mail: fabricio.gualdani@unesp.br

Francisco de Assis Noberto Galdino de Araújo

ORCID: https://orcid.org/0000-0003-1190-1923

Universidade Federal do Rio Grande do Norte, Brasil

E-mail: francisco.galdino@ufrn.br

\begin{abstract}
Resumo
Considerando as relações estabelecidas entre a Ciência da Informação e o campo da Saúde, bem como considerando que a informação em saúde é um bem comum da sociedade, este estudo buscou mapear iniciativas que tratassem disciplinas sobre informação em saúde no ensino de graduação a fim de delinear uma abordagem para o desenvolvimento de disciplinas sobre essa temática. Para tanto, desenvolveu-se uma pesquisa exploratória, para investigar disciplinas de graduação sobre informação em saúde e ofertadas por instituições de ensino superior. Para tanto, foram selecionadas três instituições de ensino superior que contemplam esta perspectiva, quais sejam: Universidade de São Paulo (Brasil), University of Victoria (Canadá) e Instituto Politécnico de Leiria (Portugal). A partir do estudo exploratório, conclui-se que é possível abordar disciplinas sobre informação em saúde em cursos de graduação, seguindo diferentes perspectivas. Este estudo propõe que sejam pensadas disciplinas sobre informação em saúde que abarquem, ao menos, temáticas sobre: a) usos da informação em saúde; b) objetos informacionais em saúde; c) comunicação em saúde; d) organização, representação e recuperação da informação no campo da saúde; e) tecnologias da informação associadas à informação em saúde. Realidades institucionais e sociais diferentes podem interferir no processo de consolidação de inovações curriculares no ensino de graduação. Logo, faz-se necessário uma reflexão aprofundada antes de qualquer alteração ou proposição curricular.
\end{abstract}

Palavras-chave: Informação em saúde; Ciência da informação; Graduação; Informática em saúde; Ensinoaprendizagem.

\begin{abstract}
Considering the relationships established between Information Science and the field of Health, as well as considering that health information is a common good of society, this study sought to map initiatives that addressed disciplines on health information in undergraduate education in order to outline an approach to the development of disciplines on this theme. Therefore, an exploratory research was developed to investigate undergraduate courses on health information offered by higher education institutions. To this end, three higher education institutions were selected that contemplate this perspective, namely: University of São Paulo (Brazil), University of Victoria (Canada) and Instituto Politécnico de Leiria (Portugal). From the exploratory study, it is concluded that it is possible to approach subjects on health information in undergraduate courses, following different perspectives. This study proposes that disciplines on health information be considered that cover, at least, themes on: a) uses of health information; b) informational objects in health; c) communication in health; d) organization, representation and retrieval of information in the health field; e) information technologies associated with health information. Different institutional and social realities can interfere in the process of consolidating curricular innovations in undergraduate education. Therefore, an in-depth reflection is necessary before any change or curricular proposal.
\end{abstract}

Keywords: Health information; Information science; Undergraduate courses; Health informatics; Teaching-learning. 


\begin{abstract}
Resumen
Considerando las relaciones establecidas entre las Ciencias de la Información y el campo de la Salud, así como considerando que la información en salud es un bien común de la sociedad, este estudio buscó mapear iniciativas que abordan asignaturas sobre información en salud en la formación de pregrado con el fin de esbozar un abordaje del desarrollo de asignaturas sobre este tema. Por lo tanto, se desarrolló una investigación exploratoria para investigar cursos de pregrado sobre información en salud ofrecidos por instituciones de educación superior. Para ello, se seleccionaron tres instituciones de educación superior que consideran esta perspectiva, a saber: Universidad de São Paulo (Brasil), Universidad Victoria (Canadá) e Instituto Politécnico de Leiria (Portugal). Del estudio exploratorio, se concluye que es posible abordar temas sobre información en salud en cursos de pregrado, siguiendo diferentes perspectivas. Este estudio propone que se consideren disciplinas sobre información en salud que abarquen, al menos, temas sobre: a) usos de la información en salud; b) objetos de información en salud; c) comunicación en salud; d) organización, representación y recuperación de información en el campo de la salud; e) tecnologías de la información asociadas a la información en salud. Diferentes realidades institucionales y sociales pueden interferir en el proceso de consolidación de las innovaciones curriculares en la educación de pregrado. Por tanto, es necesaria una profunda reflexión ante cualquier cambio o propuesta curricular.
\end{abstract}

Palabras clave: Información en salud; Ciencias de la información; Cursos de pregrado; Informática de la salud; Enseñanza-aprendizaje.

\title{
1. Introdução
}

A informação é um insumo básico no contexto evolutivo da sociedade, estando presente e sendo requerida em todo o ciclo de vida de comunidades, grupos e indivíduos. Neste sentido, a necessidade humana de externalizar e materializar em diversos suportes emoções, pensamentos, descobertas cotidianas e científicas, tem balizado a constituição e o desenvolvimento da Ciência da Informação.

Consolidada no período pós-guerra, a Ciência da Informação tem perseguido o objetivo de estudar as propriedades, comportamentos, fluxos e os meios de processamento da informação (Silva, 2006). Assumindo uma natureza colaborativa e interdisciplinar, este campo tem ainda estabelecido inúmeras aproximações com diversos campos tradicionais como a Filosofia, a Sociologia, a História, o Direito, a Medicina e a Ciência da Computação, bem como com campos emergentes como a Ciência de Dados e a Inteligência Artificial (Bartlett \& Dalkir, 2020).

No que tange aos relacionamentos entre a Ciência da Informação e o campo da Saúde, pode-se afirmar que são variados os registros que refletem essa conexão, como a criação da National Library of Medicine dos Estados Unidos em 1836 e a fundação da Medical Library Association em 1898, duas importantes instituições que até a presente data desenvolvem iniciativas voltadas a todo o ciclo informacional no campo da saúde, incluindo o desenvolvimento de acervos e tecnologias afins, o fomento a projetos de pesquisa e a formação de recursos humanos em temas relacionados à informação em saúde.

Igualmente, tem-se eventos importantes que vêm estabelecendo a conexão entre a Ciência da informação e a Saúde, como o International Congress on Medical Librarianship (ICML), cuja primeira edição ocorreu em 1953, em Londres, sendo organizado pela International Federation of Library Associations and Institutions (IFLA), World Health Organization (WHO) e associações de bibliotecas médicas. Curiosamente, em sua primeira edição, o ICML teve como discussões a era précomputador, a educação, administração e operações bibliotecárias, o alto custo dos periódicos e a história da medicina (Crawford, 1995), evidenciando as dimensões interdisciplinares da área. Atualmente, já em sua 13ª edição, o ICML1 encontrase agendado para 2022, na África do Sul, em parceria com a Association for Health Information and Libraries in Africa (AHILA), com o objetivo de refletir sobre as contribuições que bibliotecários médicos e outros profissionais da informação estão fazendo para alcançar objetivos globais de saúde, conectando-se, engajando-se, melhorando o estado de saúde dos indivíduos e salvando vidas por meio da informação em saúde.

No âmbito europeu, destaca-se também, em 1991, a criação da Associação Portuguesa de Documentação e Informação em Saúde (APDIS) cujo objetivo é dar suporte aos profissionais da informação no desenvolvimento da

${ }^{1}$ International Congress on Medical Librarianship. Disponível em: https://icml2022.org/. Acesso em: 03 dez. 2021. 
documentação e informação de saúde em Portugal e sua articulação com sistemas ou redes nacionais e internacionais, de modo a contribuir para a investigação, formação profissional e na prestação de cuidados de saúde em Portugal (Associação Portuguesa de Documentação e Informação de Saúde [APDIS], 1991).

No contexto científico brasileiro, a interdisciplinaridade entre a Ciência da Informação e a Ciência da Saúde tem um marco significativo em 1967, ano da fundação da Biblioteca Regional de Medicina (BIREME), atualmente denominada pelo termo Centro Latino-Americano e do Caribe de Informação em Ciências da Saúde, tendo como ponto de partida a "demanda crescente de literatura científica atualizada por parte dos sistemas nacionais de saúde e das comunidades de pesquisadores, profissionais e estudantes" (Centro Latino-americano e do Caribe de Informação em Ciências da Saúde [BIREME], 2007, p. 4).

Outro marco relevante no cenário brasileiro é a criação, em 1969, da Associação dos Profissionais de Informação e Documentação em Ciências da Saúde do Estado do Rio de Janeiro - APCIS/RJ, cujo objetivo tem se voltado à educação continuada de profissionais da informação que atuam no campo da saúde, organizando cursos, seminários e congressos com grande regularidade (Rede de Bibliotecas e Centros de Informação em Arte do Estado do Rio de Janeiro [Redarte], 2021).

No que diz respeito aos eventos em território brasileiro e latino americano, destaca-se, em 1992, a realização da primeira edição do Congresso Regional de Informação em Ciências da Saúde (CRICS), tendo em vista inovações em discussões científicas entre o campo da informação e comunicação científica em saúde (Amadei, 2018).

Observa-se que nos anos 2000 os eventos científicos sobre informação em saúde ganham maior destaque e periodicidade. De acordo com Sales et al. (2018), os estudos sobre informação e saúde começaram a ser publicados de forma regular na "Revista Eletrônica de Comunicação, Informação \& Inovação em Saúde" (2007), nos eventos científicos Colóquio Internacional "A Medicina na Era da Informação" (Medinfor, 2008) e Seminário Internacional de Informação para a Saúde (Sinforgeds, 2009), e na formação do Grupo de Trabalho "Informação e Saúde" (GT 11) do XI Encontro Nacional de Pesquisa em Ciência da Informação.

Além dos relacionamentos concretos entre o campo da Ciência da Informação e o campo da Saúde estabelecidos historicamente, é importante também refletir sobre a dimensão teórica do termo informação em saúde.

Com base em Moreno et al. (2009), o termo informação em saúde teve sua terminologia definida mais precisamente no século XIX juntamente com a evolução dos estudos de epidemiologia e estatísticas em saúde. Em suma, estes estudos investigaram fatores de saúde da população a fim de traçar a sua frequência num contexto coletivo. Assim, havendo a necessidade de comunicação das questões relacionadas à saúde, ao cuidado e a doença da população, impulsionaram a disseminação da informação em saúde, a qual com o tempo foi ganhando outros desdobramentos importantes para traçar políticas públicas, pesquisas acadêmicas, aproximação com a sociedade, desenvolvimentos e estruturação de sistemas de saúde.

French-Lawyer et al. (2020) compreendem a informação em saúde, em sua dimensão mais social, como aquela informação que pode ser aplicada pelo indivíduo para melhor compreender o seu estado atual de saúde ou um diagnóstico que lhe foi fornecido. Liu et al. (2020) reiteram essa ideia, afirmando que um indivíduo, ao obter e traduzir informação em conhecimento, torna-se capaz de preservar o seu estado de saúde de uma forma que seja apropriada para o seu contexto individual perante a comunidade da qual o indivíduo pertence. Svalastog et al. (2017) contextualizam a informação em saúde no conjunto de insumos pertencentes a uma sociedade digital da qual estamos inseridos.

Considerando as relações estabelecidas entre a Ciência da Informação e o campo da saúde, bem como considerando que a informação em saúde é um bem comum da sociedade, este estudo buscou mapear iniciativas que tratassem conteúdos sobre informação em saúde no ensino de graduação a fim de delinear uma abordagem para o desenvolvimento de disciplinas sobre essa temática. 


\section{Metodologia}

Este estudo partiu inicialmente de um levantamento bibliográfico exploratório sobre instituições de ensino que ofertam disciplinas de graduação focadas em informação em saúde, no cenário nacional e internacional. Ao perceber que muitos artigos faziam relatos isolados de experiências de cursos de curta duração, optou-se por considerar instituições do conhecimento dos pesquisadores que ofertam disciplinas de graduação em informação em saúde. Constituiu-se assim uma amostra de conveniência, onde foram selecionadas pelos pesquisadores três instituições que ofertam disciplinas de graduação em informação em saúde, quais sejam: Universidade de São Paulo (Brasil), University of Victoria (Canadá) e Instituto Politécnico de Leiria (Portugal), conforme apresentado no Quadro 1.

Quadro 1. Corpus da pesquisa: Instituições, cursos e disciplinas analisadas.

\begin{tabular}{|l|l|l|l|}
\hline Instituição & $\begin{array}{l}\text { Universidade de São Paulo } \\
\text { (Brasil) }\end{array}$ & University of Victoria (Canadá) & $\begin{array}{l}\text { Instituto Politécnico de Leiria } \\
\text { (Portugal) }\end{array}$ \\
\hline Disciplinas estudadas & $\begin{array}{l}\text { Disciplinas sobre informação em } \\
\text { saúde disponibilizadas para os } \\
\text { Cursos de Graduação em Ciência } \\
\text { da Informação e Informática } \\
\text { Biomédica }\end{array}$ & $\begin{array}{l}\text { Disciplinas sobre informação em } \\
\text { saúde disponibilizadas para o } \\
\text { curso de Bacharelado em Health } \\
\text { Information Science }\end{array}$ & $\begin{array}{l}\text { Disciplinas sobre informação em } \\
\text { saúde disponibilizadas curso em } \\
\text { Licenciatura em Ciências da } \\
\text { Informação em Saúde }\end{array}$ \\
\hline
\end{tabular}

Fonte: Elaborado pelos autores (2022).

Definidas as instituições que seriam objeto de estudo, buscou-se adicionalmente por documentos institucionais, conteúdos de disciplinas ofertadas, suas ementas, conteúdos de aulas, bibliografias disponíveis na Web e contatos por email para obtenção de informações e documentos adicionais.

Conforme observa-se neste percurso metodológico, este estudo é uma pesquisa exploratória, em que se realizou um estudo de caso para um aprofundamento temático, em que foi aplicado o método comparativo para investigar as diferenças e semelhanças entre as disciplinas de graduação sobre informação em saúde ofertadas por três instituições de ensino superior (GIL, 2019). Adicionalmente, é importante ressaltar que pesquisas de caráter qualitativo podem se valer da coleta de informações e dados em diferentes documentos públicos ou privados, como jornais, relatórios, cartas, e-mails e diários (CRESSWEL, 2009), como, efetivamente, foi realizado neste estudo onde inclusive se consultou várias ementas de curso e projetos pedagógicos.

\section{Resultados}

Serão apresentados a seguir as características das disciplinas sobre informação em saúde desenvolvidas pelas instituições estudadas.

\subsection{Universidade de São Paulo: disciplinas em informação em saúde}

Fundada em 1934, ao longo de sua história, a Universidade de São Paulo tem se destacado no cenário brasileiro e internacional nos diferentes campos do conhecimento. Nas duas últimas décadas, especialmente, esta Universidade tem priorizado de forma mais significativa o atendimento de demandas sociais, por meio, por exemplo, da ampliação de vagas e de outras ações afirmativas.

Nesse contexto, por volta dos anos 2000, o Departamento de Medicina Social da Faculdade de Medicina de Ribeirão Preto da Universidade de São Paulo participou da criação de novos cursos, no campus de Ribeirão Preto, como o Curso de Graduação em Informática Biomédica, o Curso de Graduação em Biblioteconomia e Ciência da Informação, Curso de Graduação em Nutrição e Metabolismo. Naquele momento histórico, o Departamento de Medicina Social idealizou cinco 
disciplinas relacionadas ao campo da informação em saúde que podem ser cursadas pelos alunos dos cursos de Informática Biomédica e Ciência da Informação e que até a presente data continuam sendo ofertadas para ambos os cursos, bem como para alunos de outros cursos interessados na temática. São elas: Fontes de Informação em Saúde, Documentação em Saúde, Comunicação e Disseminação da Informação em Saúde, Terminologias em Saúde e Tecnologias de Informação em Saúde (Galvão, 2019; Universidade de São Paulo, 2020, 2021).

A criação, implantação e consolidação dessas disciplinas sobre informação em saúde demandou vários desafios, como a contratação de docentes para ministrá-las, oferta de turmas em horário compatível para diferentes cursos e conteúdo que pudesse ser cursado por alunos em diferentes momentos da graduação, sem que houvesse pré-requisitos (Galvão, 2019).

Embora as disciplinas sobre informação em saúde sigam evoluindo com o tempo e com as necessidades acadêmicas e sociais, seus conteúdos permanecem relativamente consolidados, conforme será exposto a seguir.

A disciplina Fontes de Informação em Saúde, com 30 horas de duração, tem por objetivo propiciar ao aluno de graduação conhecimento sobre o acesso, metodologias de uso e de avaliação de conteúdo informacional disponível em bases de dados bibliográficas acadêmicas e bases de evidência em saúde com foco em profissionais da saúde e aquelas bases de dados de evidência cujo foco recai sobre a população leiga. A disciplina apresenta, inicialmente, os diferentes usuários e usos das informações e das evidências em saúde. Nesse momento, apresentam-se as principais características das necessidades informacionais da população em geral, dos pacientes, dos profissionais clínicos, dos gestores, dos pesquisadores e dos estudantes do campo da saúde. Em seguida, a disciplina trabalha com a simulação de busca de informação ou de evidências em saúde considerando casos reais de necessidade informacional (Galvão, 2011a, 2019, 2021a, 2021b). Entre as atividades realizadas na disciplina, os alunos desenvolvem projetos de revisão de literatura no âmbito da saúde seguindo alguns princípios das revisões sistemáticas, como definição da questão da revisão, mapeamento da terminologia a ser empregada, estabelecimento de estratégias avançadas de busca, e acesso e organização das referências bibliográficas (Galvão \& Ricarte, 2019). Em todas as atividades realizadas na disciplina, os alunos recebem feedbacks individuais e coletivos a fim de verificarem potenciais desvios no processo de busca informacional. A última oferta da disciplina ocorreu em 2021, na modalidade à distância com aulas síncronas, dada a pandemia de COVID-19. Há previsão de oferta da disciplina em 2022, contudo sem uma definição da modalidade de ensino (se à distância ou se presencial), em decorrência das incertezas advindas da referida pandemia, agora, já em sua quarta onda.

A disciplina Documentação em Saúde possui 30 horas de duração e tem por objetivo apresentar as várias dimensões do prontuário do paciente, incluindo: prontuário do paciente e seu histórico; prontuário do paciente e assistência em saúde; prontuário do paciente e legislação; prontuário do paciente e seus usos no ensino e na pesquisa; prontuário do paciente, superestruturas textuais e conteúdos informacionais; e prontuário eletrônico do paciente: estado da arte e perspectivas no Brasil e no mundo (Galvão, 2011b, 2021c). Em uma primeira etapa, relaciona-se o prontuário do paciente com o conceito de saúde e com o sistema de saúde. Numa segunda etapa, apresentam-se os aspectos informacionais, conteúdos obrigatórios para registro, aspectos legais, requisitos para informatização do prontuário do paciente como sua segurança e formas de acesso, sua relação com a gestão em saúde e os indicadores de saúde/doença. Numa terceira etapa, apresentam-se aspectos mais técnicos do prontuário eletrônico do paciente como padronização de registro, interoperabilidade, escalabilidade, iniciativas brasileiras de informatização do prontuário do paciente, iniciativas internacionais de informatização do prontuário do paciente e aplicativos disponibilizados nos diferentes países (Galvão \& Ricarte, 2017). A última oferta da disciplina realizada em 2021 se deu na modalidade à distância com aulas síncronas, sendo abordadas iniciativas de informatização do prontuário do paciente em países da América do Norte e América do Sul, Europa, África e Oceania (Galvão, 2021c). Há previsão de nova oferta da disciplina em 2022, contudo sem uma definição da modalidade de ensino.

Antes da pandemia de COVID-19, a disciplina Comunicação em Saúde, também com 30 horas de duração, priorizava 
a comunicação no contexto acadêmico, a comunicação com gestores e a comunicação com o público em geral. Em decorrência da pandemia, o conteúdo precisou ser mais resumido, e o conteúdo sobre comunicação com gestores precisou ser subtraído da disciplina. A abordagem da disciplina abarca exercícios práticos de comunicação oral com os diferentes públicos, comunicação em redes sociais e a comunicação para fins acadêmicos e técnicos científicos, como a comunicação oral em eventos acadêmicos. No caso da comunicação com o público em geral, a disciplina prioriza a comunicação por meio da linguagem simples, incluindo a possibilidade de contação de histórias. Os exercícios realizados são apresentados pelos alunos em sala de aula presencial ou síncrona e todos os participantes da disciplina podem emitir feedbacks sobre a efetividade das comunicações produzidas. A próxima oferta desta disciplina será em 2022 e já consta no site institucional a previsão de oferta na modalidade à distância (Galvão, 2011c, 2019, 2022).

O conhecimento das terminologias em saúde é uma das competências necessárias para a atuação profissional no campo da saúde, na prática clínica direta, para o registro da assistência prestada no prontuário do paciente ou nos sistemas institucionais de informação em saúde, assim como é requerida em situações que demandam a produção, a organização, a disseminação, a recuperação ou o intercâmbio de informações em saúde. Nessa linha, a disciplina Terminologias em Saúde, com 60 horas de duração, tem por objetivo capacitar o aluno para compreensão das linguagens de especialidade em saúde e sua relação com a organização, representação e recuperação da informação em saúde. Nas aulas iniciais da disciplina, são apresentados alguns conceitos sobre a ciência da terminologia, bem como as diferenças entre a linguagem de especialidade e a linguagem geral. Uma vez que os alunos tenham compreensão do que venha a ser uma terminologia em saúde, são abordadas algumas terminologias em saúde de relevância nacional e internacional, como é o caso da Classificação Internacional de Doenças e Problemas Relacionados à Saúde (CID), da Tabela de Procedimentos do Sistema Único de Saúde do Brasil, da Systematized Nomenclature of Medicine Clinical Terms (SNOMED-CT), da Classificação Internacional de Atenção Primária, Classificação Internacional das Deficiências, Incapacidades e Desvantagens, Classificação Internacional da Prática de Enfermagem, Descritores em Ciências da Saúde (DECS). A disciplina se desenvolve por meio de aulas teóricas, exercícios de utilização de terminologias em saúde em forma de simulação de casos clínicos ou situações clínicas ou acadêmicas reais, visitas em alguns ambientes de uso de terminologias em saúde e palestras ou entrevistas com profissionais que atuam em diferentes campos da saúde. Nas aulas teóricas e para a elaboração dos exercícios de simulação, a disciplina transcorre dentro do laboratório de informática biomédica, onde os alunos podem acessar as 7 diferentes terminologias em formato digital. As terminologias priorizadas na disciplina seguem as diretrizes brasileiras de interoperabilidade (Portaria nº 2073, 2011; Galvão, 2011d, 2021). Sua última oferta foi em 2020, sendo prevista nova oferta em 2022, contudo sem uma definição da modalidade de ensino.

A disciplina Tecnologias de Informação em Saúde, com 60 horas de duração, busca desenvolver protótipos que venham atender as necessidades de assistência em saúde da população. A disciplina se desenvolve por meio de diálogos, rodas de conversa em grupos com no máximo 5 alunos, relatos de experiências vividas que precisam de soluções, reflexões sobre os problemas enfrentados pelas populações mais carentes e vulneráveis. A partir dessas discussões, os alunos formam grupos interdisciplinares e constroem projetos de inovação tecnológica que: 1) sejam soluções para os problemas abordados ao longo das discussões, reflexões e rodas de conversa; 2) empreguem tecnologias da informação e comunicação; 3) possuam um caráter de inovação frugal e responsável, ou seja, tenha baixo custo, possa atender as camadas mais pobres da população e não gere riscos para as futuras gerações. Nessa disciplina, o docente atua como um guia das ações discentes, conforme exposto por Galvão (2011e, 2019). A última oferta dessa disciplina ocorreu em 2021, havendo previsão de oferta para 2022, também sem uma definição da modalidade de ensino. 


\subsection{University of Victoria: curso de bacharelado em informação em saúde}

O curso de Bacharelado em Health Information Science é ministrado na University of Victoria, situada em Victoria, capital da província canadense Columbia Britânica. Esta Universidade, fundada em 1903, conta em sua estrutura com nove faculdades: Administração, Educação, Engenharia e Ciência da Computação, Belas Artes, Desenvolvimento Humano e Social, Humanidades, Direito, Ciência e Ciências Sociais (University of Victoria, 2021).

Mantido pela School of Health Information Science, localizada na Faculty of Human and Social Development, o curso de bacharelado foi criado em 1981, sendo considerado como um dos mais antigos e pioneiros programas de graduação, exercendo grande influência internacional no ensino de informática em saúde. Esta mesma escola possui um programa de pósgraduação em informática em saúde e uma especialização que relaciona a informação em saúde com a Ciência da Computação (Kushniruk et al., 2006).

Neste sentido, diferente da visão de Ciência da Informação desenvolvida no contexto brasileiro, este curso segue uma vertente voltada para o desenvolvimento de sistemas, priorizando o uso das tecnologias de informação e comunicação, capazes de gerir todo o processo de produção, tratamento, disseminação e armazenamento da informação. Esta perspectiva parece relevante pois desde sempre a informação possui conexão com as tecnologias que permitem produzi-la ou usá-la, sendo atualmente a informação e as tecnologias da informação e comunicação objetos interdependentes e, a depender do contexto, indissociáveis, como no caso da saúde.

O curso de Bacharelado em Health Information Science tem como objetivo principal relacionar o campo tecnológico ao universo das ciências da saúde, possuindo uma duração de quatro anos em tempo integral, sendo composto por disciplinas das ciências da saúde, ciências exatas e ciências tecnológicas.

A sua estrutura curricular se fundamenta no modelo curricular proposto pelo Grupo de Interesse Especial em Biomédica Informática da Association for Computing Machinery, (ACM), em 1979, ou seja, seguindo as diretrizes de um modelo adotado pelos programas da área de "Computação em Saúde". Além deste currículo base, o curso também recebeu influências do modelo curricular da referida ACM sobre "Gestão de Sistemas de Informação", bem como, o Currículo Heidelberg/Heilbronn (Protti \& Fisher, 1996). Desta maneira, os esforços empreendidos na concepção de uma matriz curricular relacionando os aspectos de computação em saúde, gestão e sistemas de informação possibilitou oferecer para a sociedade canadense profissionais habilitados a trabalharem na administração de recursos de saúde, em computação educativa, em pesquisa biomédica laboratoriais e uso da saúde em sistemas de informação de cuidados (Kushniruk et al., 2006).

O primeiro ano de curso é composto por disciplinas como: Introdução à Análise de Informação em Saúde, Leitura e Escrita Acadêmica, Introdução à Tecnologia da Informação em Saúde, Programação para Saúde, Introdução aos Sistemas Canadenses de Saúde, Matemática Finita, Biologia Moderna, Anatomia Humana e Bioquímica e Saúde Humana. No segundo ano, são ofertadas as disciplinas Princípios de Design de Banco de Dados de Saúde, Introdução à Prática Profissional, Comportamento Organizacional e Gestão da Mudança, Gerenciamento e Desenvolvimento de Banco de Dados para Sistemas de Saúde, Modelos Organizacionais de Prestação de Serviços de Saúde, Fundamentos Biomédicos, Termo de Trabalho Cooperativo-1, Estatísticas para Negócios e Estatísticas para Ciências da Vida. No terceiro ano, são disponibilizadas as disciplinas de Introdução à Análise de Sistemas, Gerenciamento de Projetos, Padrões de Informação em Saúde, Aspectos Humanos dos Sistemas de Informação de Saúde, Epidemiologia, Saúde da População e Saúde Pública, Termo de Trabalho Cooperativo-2, Registros Eletrônicos e Sistemas de Apoio à Decisão, Questões Legais e de Segurança em Informática em Saúde, Redes, Interoperabilidade e Segurança de Sistemas, Aquisições de Tecnologia da Informação, e Metodologias Clínicas. Por fim, no quarto ano são oferecidas as disciplinas de Termo de Trabalho Cooperativo-3, Gestão e Tecnologia da Informação, Implicações Sociais da Tecnologia da Informação, Projeto de Sistema de Informação em Saúde, Avaliação do Sistema e Melhoria da Qualidade, e Tendências em Informática em Saúde. Integram ainda o curso dezenas de disciplinas eletivas de 
diversos campos do conhecimento como Linguística, Filosofia, Ética, Ciências Sociais Aplicadas, Administração, etc (University of Victoria, 2022a).

Embora este curso seja bem completo tanto na proposição de disciplinas obrigatórias quanto na proposição de disciplinas eletivas, chama a atenção em sua constituição, várias disciplinas específicas sobre informação em saúde que podem ser norteadoras para a proposição de disciplinas neste campo no Brasil e que serão apresentadas a seguir.

A disciplina Introdução à Análise de Informação em Saúde explora uma variedade de abordagens e métodos emergentes para análise de dados de saúde para melhorar a saúde, sendo detalhadas a preparação e integração de dados de saúde em uma variedade de sistemas de informação. A aplicação de métodos de análise de dados para melhorar os processos de saúde em uma variedade de configurações é descrita, incluindo aplicações clínicas e uso de dados de saúde para a prática de informática em saúde, pesquisa e educação (University of Victoria, 2022b).

A disciplina Introdução à Tecnologia da Informação em Saúde aborda os sistemas de informação em saúde compostos por programas de computador gerados por meio de uma variedade de técnicas de manipulação e gerenciamento de dados. Abrange a aplicação geral de bancos de dados e planilhas para gerenciamento de informações de saúde. Além disso, são abordados muitos aplicativos específicos de saúde, como registros eletrônicos de saúde, aplicativos móveis de saúde, gráficos médicos, sistemas de informação médica multimídia, sistemas de informação de saúde pública, sistemas de apoio à decisão, sistemas em rede, aplicativos de saúde vestíveis e bioinformática (University of Victoria, 2022c).

A disciplina Padrões de Informação em Saúde aborda os padrões de informação em saúde implantados e usados no Canadá e em outros países, entre os quais os padrões terminológicos com a CID e o SNOMED CT. São estudados a natureza dos padrões, sua evolução histórica e os ciclos de vida, desde o desenvolvimento inicial, distribuição até manutenção, enfatizando-se o conhecimento prático e as habilidades necessárias para se trabalhar com padrões (University of Victoria, 2022d).

A disciplina Aspectos Humanos dos Sistemas de Informação de Saúde abarca aspectos humanos da informática em saúde, incluindo a discussão sobre o uso de tecnologia para apoiar a tomada de decisão clínica e diminuir a incidência de erros nos cuidados de saúde. Além disso, discute a recuperação de informações e os processos cognitivos envolvidos, tomada de decisão e raciocínio clínico, interação humano-computador, usabilidade, segurança do paciente e redução de erros, medicina baseada em evidências, mHealth, informática do consumidor, literacia em e-saúde e análise de fluxo de trabalho (University of Victoria, 2022e)

A disciplina Registros Eletrônicos e Sistemas de Apoio à Decisão oferece aos alunos uma compreensão profunda das tecnologias que compõem os registros eletrônicos de saúde e os sistemas de apoio à decisão, incluindo registros eletrônicos de saúde (de código aberto e comerciais), sistemas de informação departamentais (laboratório, farmácia, terapia intensiva), arquivamento de imagens e sistemas de comunicação, prescrição eletrônica de saúde, repositórios de dados clínicos, portais de pacientes e registros pessoais de saúde (University of Victoria, 2022f).

A disciplina Questões Legais e de Segurança em Informática em Saúde apresenta aspectos legais, incluindo aspectos de confidencialidade, privacidade, responsabilidade legal de sistemas de software, questões contratuais, terminologia legal, raciocínio e processos legais, princípios legais que se aplicam e governam os sistemas de saúde, bem como estratégias de segurança cibernética em saúde (University of Victoria, 2022g).

A disciplina Redes, Interoperabilidade e Segurança de Sistemas examina o impacto da tecnologia de comunicação nos sistemas de informação em vários setores do sistema de saúde, incluindo redes de registros eletrônicos de saúde, interoperabilidade e padrões clínicos, aspectos técnicos de cibersegurança, telessaúde, monitoramento de assistência médica domiciliar e computação difusa em assistência médica (University of Victoria, 2022h).

A disciplina Aquisições de Tecnologia da Informação apresenta metodologias e processos usados para selecionar tecnologias da informação para organizações de saúde e governos. Avalia a dinâmica e os compromissos firmados, 
especialmente, quando as organizações de saúde e os governos adquirem TI para apoiar os pacientes e o público, incentivando a pensar sob o ponto de vista clínico, bem como do ponto de vista técnico e de gestão (University of Victoria, 2022i).

A disciplina Metodologias Clínicas aborda o processo de tomada de decisão clínica, contemplando o uso de sistemas eletrônicos na melhoria do processo de tomada de decisão clínica, bem como, a prevenção de erros de diagnóstico, integração da medicina baseada em evidências e diretrizes clínicas, melhoria da tomada de decisão baseada em equipe e fatores que afetam a tomada de decisão nas políticas públicas (University of Victoria, 2022j).

A disciplina Gestão e Tecnologia da Informação possibilita identificar estratégias e abordagens para garantir que gerentes, médicos, pacientes e o público recebam informações na hora certa e de maneira econômica (University of Victoria, 20221).

A disciplina Implicações Sociais da Tecnologia da Informação reflete sobre os aspectos sociais e éticos das tecnologias de informação em saúde, analisando algumas questões sistêmicas e sociais relacionadas à revolução dessas tecnologias e seus impactos na condição humana individual e coletiva (University of Victoria, 2022m).

Embora o curso de Bacharelado em Health Information Science da University of Victoria possua muitos conteúdos que enfatizem o uso das TICs e as atividades gerenciais em sistemas de informação em saúde, percebe-se relações entre estas disciplinas com os aspectos fundamentais da Ciência da Informação. Relação esta que possibilita observar a Informação em Saúde em um contexto amplo de arquitetura e usabilidade da informação em sistemas, bem como, o papel da informação no contexto "homem-sociedade".

\subsection{Instituto Politécnico de Leiria: curso de licenciatura em ciências da informação em saúde}

O Instituto Politécnico de Leiria, em uma parceria entre a Escola Superior de Saúde e a Escola Superior de Tecnologia e Gestão, criou o curso em Licenciatura em Ciências da Informação em Saúde no ano de 2015, a partir da reformulação do curso em Licenciatura em Informática para a Saúde, com o objetivo de lançar profissionais, em meio a falta no mercado de trabalho e com a inovação/informatização do Sistema de Saúde de Portugal nos últimos anos, que tivessem a especialização para atuarem na área de Saúde com conhecimentos também na área de Informática, Ciência da Informação, Estatística e Ciências Sociais.

O Instituto Politécnico de Leiria é uma instituição pública portuguesa de ensino superior que iniciou suas atividades em 1980, composto por cinco escolas localizadas na região de Leiria e Oeste, sendo elas: Escola Superior de Educação e Ciências Sociais, Escola Superior de Tecnologia e Gestão e Escola Superior de Saúde (localizadas nas cidades de Leiria), Escola Superior de Artes e Design (em Caldas da Rainha) e Escola Superior de Turismo e Tecnologia do Mar (em Peniche).

O Instituto possui cursos nas modalidades: Técnico Superior Profissional (duração de quatro semestres) corresponde a cursos de nível pós-secundário e não superior, tem um caráter formativo técnico e para o contexto de trabalho; Licenciatura, sendo um curso em ensino superior com duração entre seis a oito semestres letivos, que aborda a questão científica e aplicação dos conhecimentos especializados na prática de trabalho; Pós-graduação, tem a caraterística de curso de especialização em determinada matéria e não confere o grau acadêmico aos estudantes; Mestrado, um curso com duração de dois a quatro semestres, confere o grau acadêmico de mestre numa determinada especialidade.

Com os incrementos das tecnologias da informação e comunicação no Sistema de Saúde de Portugal, intensificados em 2016, a fim de haver uma maior troca informacional entre os órgãos de Saúde e os cidadãos e uma maior eficiência do Sistema de Saúde, foram implementados no Sistema Nacional de Saúde tecnologias para a inovação de seus serviços e sistemas, tais como a telemedicina. A promoção do uso da tecnologia da informação difundida pelo Plano Nacional de Saúde português é reforçada pelo Ministério da Saúde principalmente em relação aos medicamentos e dispositivos médicos e terapêuticos (Matos \& Nunes, 2018). 
Desse modo, a adesão do Sistema de Saúde português à estratégia de e-Health permitiu uma maior integração da informação, produção, armazenamento, consulta, difusão, coleta/acesso de dados e gestão de recursos, por meio do uso das tecnologias da informação na saúde, tornando os processos mais eficientes, eficazes, econômicos e confiáveis.

Desse modo, para atender essa inovação tecnológica no âmbito dos serviços de saúde, voltado para uma formação técnica e prática, o curso tem a proposta de capacitar o aluno com habilidades e competências para o mercado de trabalho, para que ele possa interpretar, buscar, visualizar, classificar, analisar, preservar e trabalhar eticamente com dados, estatísticas, informações voltadas para a área de Saúde e o Sistema de Saúde e concomitantemente ter a aptidão para uso e desenvolvimento de Tecnologias e Sistemas de Informação (TSI). O uso da tecnologia tem como objetivo ser um modo de apoio e auxílio à saúde pública, às gestões clínicas e hospitalares, à organização das informações eletrônicas de prescrição de medicamentos, diagnósticos, terapêutica, exames, entre outras relacionadas aos cuidados com os pacientes.

As atividades práticas do curso são caracterizadas pelo desenvolvimento de projetos, estudos e estágio realizados em parceria com organizações de Saúde e unidades de investigação. De acordo com o plano de estudos, a partir do segundo ano, os discentes devem realizar a disciplina de Projeto, podendo ser desenvolvida internamente nas unidades curriculares ou em unidades externas como em organizações públicas ou privadas, a qual eles são estimulados a organizar e documentar seus trabalhos, como a elaboração de documentos como manuais e relatórios finais, a fim de resolver problemas e criar soluções para estes. Para isso, os alunos são inseridos em grupos, com a supervisão de dois docentes da área de Saúde e Informática, devem saber propor iniciativas, inovações e trabalhar em equipe. (Despacho n. $.^{\circ} 8484 / 2015$, 2015; Regulamento n. ${ }^{\circ}$ 566/2018, 2018; Regulamento n. ${ }^{\circ} 572 / 2018,2018$ ).

Em relação ao estágio, este é um processo em que o aluno tem o contato com os locais de prática, a fim de desenvolver habilidades para o trabalho com as TSI em contextos ligados à saúde. Nesse processo, o aluno possui a supervisão de docentes do curso da equipe de estágio e de um profissional que orienta seu trabalho na entidade de estágio, com o objetivo de integrar os conhecimentos do curso à sua aplicação prática em um ambiente organizacional. Assim, o discente deverá devolver competências para a tomada de decisões, trabalho em equipe, entre outras.

O curso tem a duração de seis semestres letivos, contemplando disciplinas sobre Saúde, Ciências Informáticas, Ciências Sociais e do Comportamento, Estatística e Línguas e Literaturas Estrangeiras. Entre elas podem ser mencionadas: Anatomia e Fisiologia; Psicossociologia da Saúde; Epidemiologia e Saúde Pública; Primeiros Socorros e Suporte Básico de Vida; Computadores e Programação; Inglês; Educação e Comunicação em Saúde; Caracterização de Sistemas e Serviços de Saúde; Plataformas de Informação de Saúde; Gestão de Dados de Saúde-1; Tecnologias de Diagnóstico; Projeto-1; Modelos de Intervenção Terapêutica; Economia da Saúde; Computadores e Programação-2; Gestão de Dados de Saúde-2; Redes de Dados de Saúde; Processos de Cuidados de Saúde; Metodologias de Desenvolvimento de Software; Laboratório de Sistemas Operativos; Aplicações Web; Desenho da Informação em Saúde; Qualidade em Saúde; Arquitetura e Tecnologia dos Computadores; Informoterapia; Projeto-2; Investigação em Saúde; Classificação e Codificação Clínica; Sistemas de Informação em Saúde; Integração de Sistemas de Informação; Privacidade e Proteção de Dados de Saúde; Estatística Aplicada à Saúde; Prática Baseada na Evidência; Bioinformática; Empreendedorismo em Saúde; Tópicos Avançados de Aplicações de Saúde; Registos Clínicos Eletrônicos; Projeto Final/Estágio.

Das disciplinas mencionadas pode-se inferir que algumas estão mais próximas da Ciência da Informação como: Gestão de dados em Saúde; Privacidade e Proteção de Dados de Saúde; Plataformas de Informação de Saúde, Educação e Comunicação em Saúde; Informoterapia; Classificação e Codificação Clínica; Registos Clínicos Eletrônicos; Prática Baseada na Evidência; Desenho da Informação em Saúde; Aplicações Web; e Redes de Dados de Saúde.

O sítio institucional do Instituto Politécnico de Leiria não disponibiliza publicamente as ementas das disciplinas do Curso de Licenciatura em Ciências da Informação em Saúde. Dessa forma, a instituição foi contatada algumas vezes entre 
novembro de 2021 e janeiro de 2022 pelo correio eletrônico institucional oficial para que se obtivesse informações mais detalhadas sobre o curso. Apenas no dia 11 de janeiro de 2022, obteve-se uma resposta oficial de que este curso teve sua oferta descontinuada. Os pesquisadores optaram por manter as informações obtidas no presente artigo para registro histórico.

\subsection{Proposta de abordagem}

Após ter mapeado iniciativas institucionais descritas anteriormente, elaborou-se o Quadro 2, onde se pode comparar as diversas disciplinas sobre informação em saúde oferecidas pelas três instituições, bem como se pode observar traços de similaridade que poderão nortear o desenvolvimento de disciplinas sobre informação em saúde em outros cursos de graduação, sendo os principais pontos de convergência temática encontrados: a) usos da informação, abarcando o acesso às fontes de informação acadêmica e clínica, bem como o emprego das informações encontradas para a prática clínica, para a pesquisa clínica e acadêmica, bem como pelos pacientes; b) objetos informacionais, contemplando, principalmente, o prontuário do paciente e suas especificidades informacionais, tecnológicas, legais e éticas; c) comunicação, abarcando processos comunicacionais com profissionais da saúde, gestores e grande público, bem como seus modos de apresentação da informação em saúde em diferentes meios; d) organização, representação e recuperação da informação, incluindo as diversas terminologias em saúde, processos de análise da informação, gestão da informação e dados, e aspectos relacionados à interoperabilidade; e) tecnologias da informação, abarcando todo o aparato tecnológico aplicado às informações e dados em saúde, sua dimensão técnica, social, política, econômica, ética e legal.

Quadro 2. Sistematização de disciplinas sobre informação em saúde encontradas nas três instituições estudadas.

\begin{tabular}{|c|c|c|c|}
\hline Convergência temática & $\begin{array}{l}\text { Universidade de São } \\
\text { Paulo }\end{array}$ & University of Victoria & Instituto Politécnico de Leiria \\
\hline Usos da informação & $\begin{array}{l}\text { Fontes de Informação em } \\
\text { Saúde }\end{array}$ & $\begin{array}{l}\text { Gestão e Tecnologia da } \\
\text { Informação } \\
\text { Metodologias Clínicas }\end{array}$ & $\begin{array}{l}\text { Prática Baseada na Evidência; } \\
\text { Plataformas de Informação de Saúde } \\
\text { Investigação em Saúde }\end{array}$ \\
\hline Objetos informacionais & Documentação em Saúde & $\begin{array}{l}\text { Registros Eletrônicos e } \\
\text { Sistemas de Apoio à Decisão }\end{array}$ & Registros Clínicos Eletrônicos \\
\hline Comunicação & Comunicação em Saúde & & $\begin{array}{l}\text { Educação e Comunicação em Saúde; } \\
\text { Desenho da Informação em Saúde }\end{array}$ \\
\hline $\begin{array}{l}\text { Organização, representação e } \\
\text { recuperação da informação }\end{array}$ & Terminologias em Saúde & $\begin{array}{l}\text { Introdução à Análise de } \\
\text { Informação em Saúde; } \\
\text { Padrões de Informação em } \\
\text { Saúde; } \\
\text { Redes, Interoperabilidade e } \\
\text { Segurança de Sistemas }\end{array}$ & $\begin{array}{l}\text { Classificação e Codificação Clínica; } \\
\text { Gestão de dados em Saúde; } \\
\text { Redes de Dados de Saúde }\end{array}$ \\
\hline Tecnologias da informação & $\begin{array}{l}\text { Tecnologias de } \\
\text { Informação em Saúde }\end{array}$ & $\begin{array}{l}\text { Introdução à Tecnologia da } \\
\text { Informação em Saúde; } \\
\text { Aquisições de Tecnologia da } \\
\text { Informação } \\
\text { Implicações Sociais da } \\
\text { Tecnologia da Informação } \\
\text { Aspectos Humanos dos } \\
\text { Sistemas de Informação de } \\
\text { Saúde; } \\
\text { Questões Legais e de Segurança } \\
\text { em Informática em Saúde }\end{array}$ & $\begin{array}{l}\text { Privacidade e Proteção de Dados de } \\
\text { Saúde }\end{array}$ \\
\hline
\end{tabular}

Fonte: Elaborado pelos autores (2022). 


\section{Conclusão}

A partir do estudo exploratório contemplando iniciativas propostas pela Universidade de São Paulo (Brasil), pela University of Victoria (Canadá) e pelo Instituto Politécnico de Leiria (Portugal), conclui-se que é possível abordar disciplinas sobre informação em saúde em cursos de graduação, seguindo diferentes perspectivas. Este estudo propõe que sejam pensadas disciplinas sobre informação em saúde que abarquem, ao menos, temáticas sobre: a) usos da informação em saúde; b) objetos informacionais em saúde; c) comunicação em saúde; d) organização, representação e recuperação da informação no campo da saúde; e) tecnologias da informação associadas à informação em saúde - conforme representado na Figura 1.

Figura 1. Proposta de abordagem para disciplinas de informação em saúde a serem oferecidas em outros cursos de graduação.

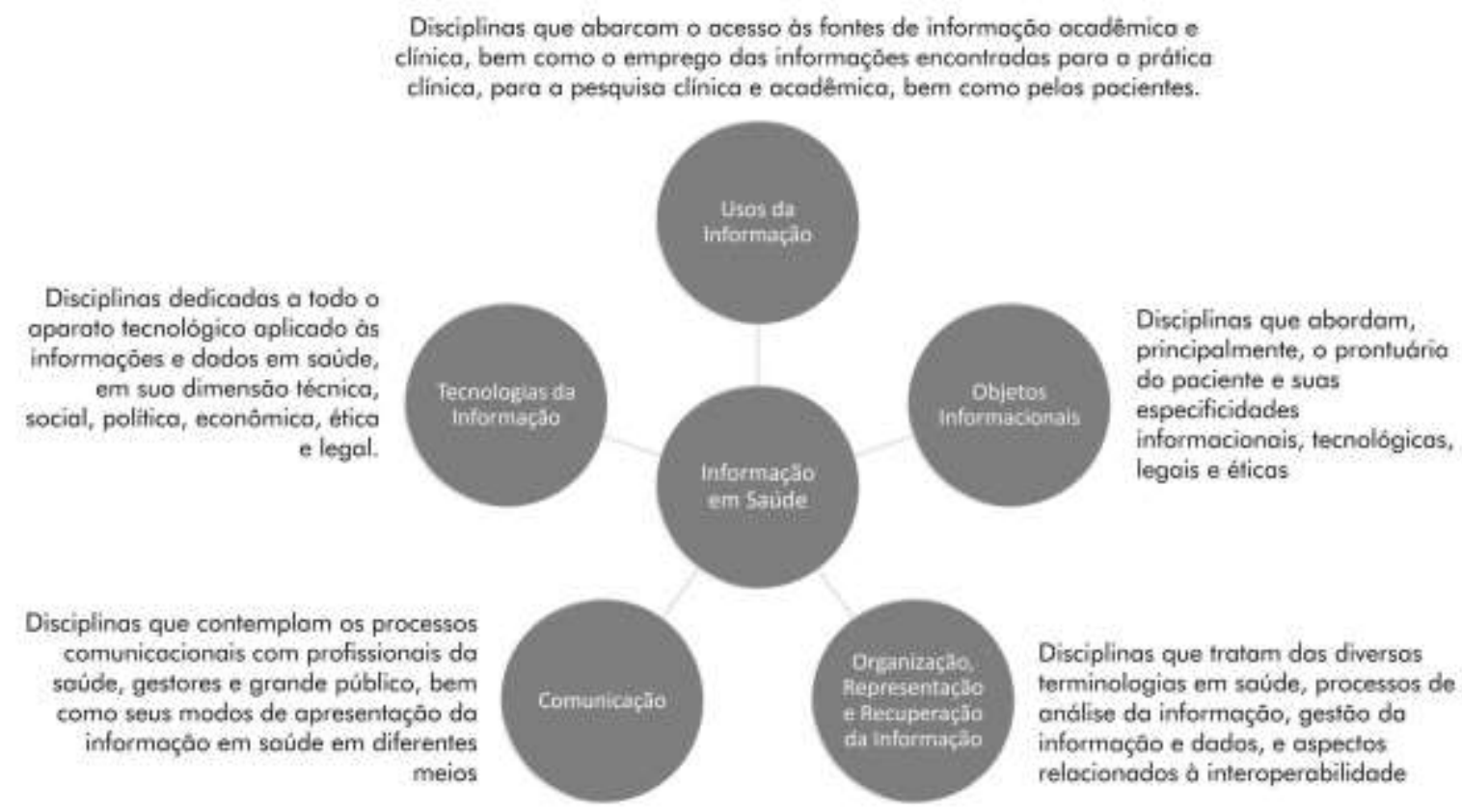

Fonte: Elaborado pelos autores (2022).

Conforme observado na Figura 1, a concepção dessa proposta não é especificar o nome de disciplinas, mas propor conteúdos que podem ser trabalhados por diferentes cursos e de diferentes formas. Por exemplo, em usos de informação podem ser disponibilizadas disciplinas sobre o uso de recursos informacionais em saúde, saúde baseada em evidências, pesquisa bibliográfica em saúde, usos de bases de dados científicas, uso de bases de evidência em saúde para fins clínicos. Logo, cada instituição de ensino superior pode e deve buscar denominações para suas disciplinas que melhor representem esse conteúdo a partir do projeto pedagógico em que estas disciplinas forem inseridas e a partir de referencial teórico que for adotado. Igualmente, a carga horária e o número de disciplinas sobre cada conteúdo podem variar de acordo com os recursos existentes em cada instituição. Logo, essa é uma proposta de caráter conceitual, que poderá ser desdobrada de várias formas para as diferentes realidades de formação profissional.

Finalmente, o fato do curso em Licenciatura em Ciências da Informação em Saúde do Instituto Politécnico de Leiria ter sido descontinuado em tão pouco tempo de existência e o Bacharelado em Health Information Science da University of Victoria se manter ativo após 40 anos de existência parece evidenciar que realidades institucionais e sociais diferentes podem interferir no processo de consolidação de inovações curriculares no ensino de graduação. Logo, faz-se necessário uma reflexão aprofundada antes de qualquer alteração ou proposição curricular. 
Por este ter sido um estudo qualitativo exploratório, recomenda-se sua futura expansão para estudos de disciplinas sobre informação e saúde que possam estar presentes em outros cursos de graduação e pós-graduação do Brasil e do exterior a fim de se mapear cenários nacionais e internacionais mais amplos e representativos.

\section{Agradecimentos}

Dedicamos este trabalho aos docentes do Departamento de Medicina Social da Faculdade de Medicina de Ribeirão Preto da Universidade de São Paulo que participaram da concepção inicial de várias disciplinas sobre Informação em Saúde nesta instituição, especialmente, ao Prof. Dr. Juan Stuardo Yazlle Rocha e a Profa. Dr. Elisabeth Meloni Vieira.

\section{Referências}

Amadei, J. R. P. (2018). CRICS 10 - Congresso Regional de Informação em Ciências da Saúde. http://sddinforma.fob.usp.br/crics-10-congresso-reginal-deinformacao-em-ciencias-da-saude/.

André; S., \& Ribeiro, P. (2020). E-health: as TIC como mecanismo de evolução em saúde. Gestão $e$ Desenvolvimento, 28, 95-116. https://doi.org/10.34632/gestaoedesenvolvimento.2020.9467.

Associação Portuguesa de Documentação e Informação de Saúde (1991). Estatutos. https://apdis.pt/estatutos/.

Bartlett, J. C., \& Dalkir, K. (2020). Librarianship and beyond: the twenty-year evolution of an interdisciplinary curriculum. Education for Information, 36(2), 139-155. http://doi.org/10.3233/EFI-190313.

Biaggi, C. de (2019). A atuação do bibliotecário na área da saúde no contexto da gestão do fluxo da informação. [Dissertação de mestrado, Universidade Estadual Paulista]. https://repositorio.unesp.br/bitstream/handle/11449/182080/biaggi_c_me_mar.pdf?sequence=3\&isAllowed=y.

Centro Latino-americano e do Caribe de Informação em Ciências da Saúde (2007). Biblioteca Virtual em Saúde: tutorial de pesquisa bibliográfica. https://funjob.edu.br/wp-content/uploads/2019/08/tutorial_bireme.pdf.

Crawford, S. (1995). The International Congress on Medical Librarianship, 1953-1995: Goals and Achievements. Health Information for Global Village: Proceedings of the 7th International Congress on Medical Librarianship, Washington, D.C. http://www.icml9.org/archives/crawf.htm.

Cresswell, J. W. (2009). Research design: qualitative, quantitative and mixed methods approaches. Sage.

Despacho n. ${ }^{\circ} 8484 / 2015$, de 03 agosto de 2015 do Instituto Politécnico de Leiria (2015). Diário da República: Série II, n. ${ }^{\circ} 149 / 2015$, 21482 - 21484. https://dre.pt/dre/detalhe/despacho/8484-2015-69927727.

Eisenhardt, A., Liu, M., \& Murphy, D. (2011). Responding to the global crisis: developing an undergraduate program in health information management. Journal of Computing Sciences In Colleges, 26(3), 106-114. https://dl.acm.org/doi/abs/10.5555/1859159.1859182.

Fisher, P., \& Protti, D. (1996). Health Informatics at the University of Victoria. Yearbook of Medical Informatics, 5(1), 135-139. https://www.thiemeconnect.com/products/ejournals/pdf/10.1055/s-0038-1638056.pdf.

French-Lawyer, J., Siano, S., Ioerger, M., Young, V., \& Turk, M. A. (2021). Health information seeking and people with disability: a systematic search and scoping review. Disability and Health Journal, 14(1), 100983. https://doi.org/10.1016/j.dhjo.2020.100983.

Galvão, M. C. B. (2011a). Disciplina Fontes de informação em saúde : ementa . https://uspdigital.usp.br/jupiterweb/obterDisciplina?nomdis=\&sgldis=rms0014.

Galvão, M. C. B. (2011b). Disciplina Documentação em saúde: ementa . https://uspdigital.usp.br/jupiterweb/obterDisciplina?nomdis=\&sgldis=rms0011

Galvão, M. C. B. (2011c, Janeiro 2022). Disciplina Comunicação e difusão de conhecimentos em saúde: ementa. https://uspdigital.usp.br/jupiterweb/obterDisciplina?nomdis=\&sgldis=rms0016.

Galvão, M. C. B. (2011d). Disciplina Terminologias em saúde: ementa. https://uspdigital.usp.br/jupiterweb/obterDisciplina?sgldis=RMS0012.

Galvão, M. C. $\quad$ B. (2011e). Disciplina Tecnologias da informação ementa. https://uspdigital.usp.br/jupiterweb/obterDisciplina?nomdis=\&sgldis=rms0017.

Galvão, M. C. B. (2019). Disciplinas sobre informação em saúde ministradas entre 2008-2018 na Universidade de São Paulo. Preprint. http://eprints.rclis.org/38440/

Galvão, M. C. B. (2021). Classificações, terminologias e ontologias no campo da saúde. Asklepion: Informação em Saúde, 1(2), 41-54. https://asklepionrevista.info/asklepion/article/view/26. Acesso em: 4 jan. 2022.

Galvão, M. C. B. (2021a). Usuários da informação em saúde: das necessidades aos produtos e serviços informacionais. In Helen de Castro Silva Casarin. (Org.), Usuários da informação e diversidade (1-27). Cultura Acadêmica.

Galvão, M. C. B. (2021b). Disciplina Fontes de informação em saúde: plano de ensino-aprendizagem. https://cg.fmrp.usp.br/pb/cursos/informaticabiomedica/planos-de-ensino-e-aprendizagem/. 
Galvão, M. C. B. (2021c). Disciplina Documentação em saúde: plano de ensino-aprendizagem. https://cg.fmrp.usp.br/pb/cursos/informaticabiomedica/planos-de-ensino-e-aprendizagem/.

Galvão, M. C. B. (2022). Disciplina Comunicação em saúde: plano de ensino-aprendizagem. https://cg.fmrp.usp.br/pb/cursos/informatica-biomedica/planosde-ensino-e-aprendizagem/.

Galvão, M. C. B., \& Ricarte, I. L. M. (2017). Alinhamentos necessários entre o registro eletrônico de saúde e o sistema de saúde. Informação \& Informação, 22(3), 426-455, 2017.https://doi.org/10.5433/1981-8920.2017v22n3p426.

Galvão, M. C. B., \& Ricarte, I. L. M. (2019). Revisão sistemática da literatura: conceituação, produção e publicação. Logeion: Filosofia da Informação, 6(1), 57-73, 2019. https://doi.org/10.21728/logeion.2019v6n1.p57-73.

GIL, A. C. (2019). Métodos e técnicas de pesquisa social. Atlas.

Kushniruk, A., Lau, F., Borycki, E., Prott, D. (2006). The School of Health Information Science at the University of Victoria: towards an integrative model for health informatics education and research. Yearbook Of Medical Informatics, 15(1), 159-165. https://www.thiemeconnect.com/products/ejournals/pdf/10.1055/s-0038-1638477.pdf.

Liu, C., Wang, D., Liu, C., Jiang, J., Wang, X., Chen, H., Ju, X., \& Zhang, X. (2020). What is the meaning of health literacy? A systematic review and qualitative synthesis. Family Medicine and Community Health, 8(2), e000351. https://doi.org/10.1136/fmch-2020-000351

Matos, A. A., \& Nunes, A. M. (2018). Tecnologias da informação e comunicação no sistema de saúde Português. Journal of Health Informatics, 10(1), 30-34. http://www.jhi-sbis.saude.ws/ojs-jhi/index.php/jhi-sbis/article/viewFile/571/328.

Moreno, A. B.; Coeli, C. M.; \& Munck, S. ([2009]). Informação em Saúde. In Dicionário da educação profissional em saúde. Fiocruz. http://www.sites.epsjv.fiocruz.br/dicionario/verbetes/infsau.html.

Murphy, J. (2021). Digital health interventions: new opportunities for health science librarians. Health Information \& Libraries Journal, 38(3), 231-236, 2021.

Portaria $\mathrm{n}^{\circ}$ 2073, de 31 de agosto de 2011 do Ministério da Saúde (2011). Regulamenta o uso de padrões de interoperabilidade e informação em saúde para sistemas de informação em saúde no âmbito do Sistema Único de Saúde, nos níveis Municipal, Distrital, Estadual e Federal, e para os sistemas privados e do setor de saúde suplementar. Diário Oficial da União: Seção 1, 63. https://bvsms.saude.gov.br/bvs/saudelegis/gm/2011/prt2073_31_08_2011.html.

Rede de Bibliotecas e Centros de Informação em Arte do Estado do Rio de Janeiro (2021). Outras redes de informação. https://www.redarte.org.br/outrasredes-de-informacao-2/.

Regulamento n. ${ }^{\circ}$ 566/2018, de 21 de agosto de 2018 do Instituto Politécnico de Leiria (2018). Diário da República: Série II, n..$^{\circ}$ 160/2018, 23336-23339. https://dre.pt/dre/detalhe/regulamento/566-2018-116127307.

Regulamento n. ${ }^{\circ}$ 572/2018, de 22 de agosto de 2018 do Instituto Politécnico de Leiria (2018). Diário da República: Série II, n. ${ }^{\circ}$ 161/2018, Portugal, 2353223534. https://dre.pt/dre/detalhe/regulamento/572-2018-116149602.

Sales, O. M. M.; Oliveira, H. P. C. de, \& Pinto, V. B. (2018). Ciência da Informação e Ciências da Saúde: diálogos construídos por meio da interdisciplinaridade. [Apresentação de trabalho]. Anais do $19^{\circ}$ Encontro Nacional de Pesquisa em Ciência da Informação, Londrina. https://brapci.inf.br/index.php/res/download/124737.

Silva, A. M. da (2006). A informação: da compreensão do fenómeno e construção do objecto científico. Editora Afrontamento.

Svalastog, A. L., Donev, D., Jahren Kristoffersen, N., \& Gajović, S. (2017). Concepts and definitions of health and health-related values in the knowledge landscapes of the digital society. Croatian Medical Journal, 58(6), 431-435. https://doi.org/10.3325/cmj.2017.58.431

Universidade de São Paulo (2020). Projeto pedagógico do Curso de Graduação em Informática Biomédica. http://cg.fmrp.usp.br/wpcontent/uploads/sites/369/2021/02/PP-IBm-atualizado-em-2020.pdf.

Universidade de São Paulo (2021). Projeto pedagógico do Curso de Bacharelado em Biblioteconomia e Ciência da Informação. https://www.ffclrp.usp.br/graduacoes/cursos.php?g=57.

University of Victoria (2021). About the University. https://www.uvic.ca/about-uvic/about-the-university/index.php.

University of Victoria (2022a). Health Information Science: Bachelor of Science - Major. https://bit.ly/3GeFZ1G .

University of Victoria (2022b). HINF112 Introduction to Health Information Analysis. https://www.uvic.ca/calendar/undergrad/index.php\#/courses/view/5f4693a16baaa900265f647e .

University of Victoria (2022c). HINF130 Introduction to Health Information Technology. https://www.uvic.ca/calendar/undergrad/index.php\#/courses/view/5e59504cf3996725007d5863

$\begin{array}{lllllll}\text { University } & \text { of } & \text { Victoria } & \text { (2022d). HINF335 } & \text { Health } & \text { Information }\end{array}$
https://www.uvic.ca/calendar/undergrad/index.php\#/courses/view/5e5950843a898c2500fbf160.

University of Victoria (2022e). HINF350 Human Aspects of Health Care Information Systems. https://www.uvic.ca/calendar/undergrad/index.php\#/courses/view/5d544ef54ff8972400f65a0b .

University of Victoria (2022f). HINF310 Electronic Records and Decision Support Systems. https://www.uvic.ca/calendar/undergrad/index.php\#/courses/view/5d1f6f92d2bc1524008cb132.

University of Victoria (2022g). HINF330 Legal and Security Issues in Health Informatics. https://www.uvic.ca/calendar/undergrad/index.php\#/courses/view/5d1f74a6d2bc1524008cb4f4 . 
Research, Society and Development, v. 11, n. 2, e49411226194, 2022

(CC BY 4.0) | ISSN 2525-3409 | DOI: http://dx.doi.org/10.33448/rsd-v11i2.26194

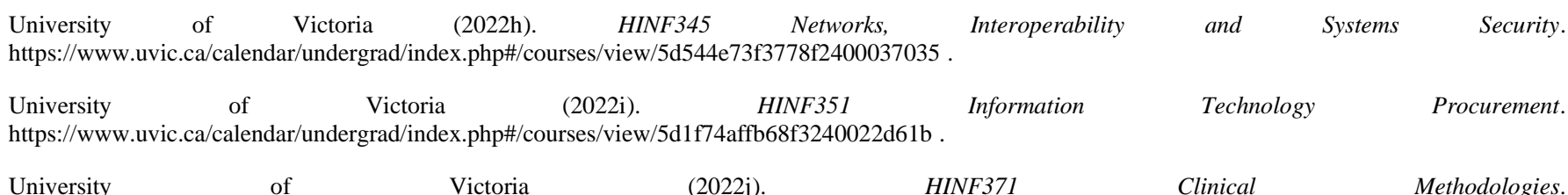
University
https://www
of
Victoria
$(2022 \mathrm{j})$

HINF371

Methodologies.

University of Victoria (20221). HINF410 Information https://www.uvic.ca/calendar/undergrad/index.php\#/courses/view/5e5951223a898c2500fbf16a .

University of Victoria $\quad(2022 \mathrm{~m}) . \quad$ Societal Implications Information of https://www.uvic.ca/calendar/undergrad/index.php\#/courses/SkH1O_pXN?bc=true\&bcCurrent=Societal\%20Implications\%20of\%20Information\%20Technolo gy\&bcGroup=Health\%20Information $\% 20$ Science $\% 20($ HINF) \&bcItemType=Courses 